\title{
Effect of Equi-Strath ${ }^{\circledR}$ on Leucocyte Occurrence in the Haired Skin of Horses
}

\author{
Danja D. Wiederkehr'1, Elise Jeannerat ${ }^{2}$, Paivi Nussbaumer², Dominik Burger ${ }^{2}$, \\ Hendrika Anette van Dorland ${ }^{1}$ \\ ${ }^{1}$ School of Agricultural, Forest and Food Sciences, Bern University of Applied Sciences, Bern, Switzerland \\ ${ }^{2}$ Swiss Institute of Equine Medicine, University of Bern, and Agroscope, Bern, Switzerland \\ Email: danja.wiederkehr@bfh.ch
}

How to cite this paper: Wiederkehr, D.D., Jeannerat, E., Nussbaumer, P., Burger, D. and van Dorland, H.A. (2018) Effect of Equi-Strath on Leucocyte Occurrence in the Haired Skin of Horses. Agricultural Sciences, 9, 1-7.

https://doi.org/10.4236/as.2018.91001

Received: November 29, 2017

Accepted: January 5, 2018

Published: January 8, 2018

Copyright $\odot 2018$ by authors and Scientific Research Publishing Inc. This work is licensed under the Creative Commons Attribution International License (CC BY 4.0).

http://creativecommons.org/licenses/by/4.0/

\begin{abstract}
Strath ${ }^{\circledast} /$ Bio-Strath $^{\oplus}$ is a plasmolyzed herbal yeast product with malt, honey and orange syrup. Its ability to support the immune system of the human being is well known and was tested in several studies. Equi-Strath ${ }^{\circ}$, the adapted product for horses was tested in this study. The aim of the study was to analyze the effects of Equi-Strath ${ }^{\otimes}$ on skin condition in the horse. The study included twenty healthy Franches-Montagnes stallions which were divided into two groups. The study group (SG, $\mathrm{n}=10$ ) received $0.06 \mathrm{ml} / \mathrm{kg}$ bodyweight of Equi-Strath ${ }^{\oplus}$, the control group $(C G, n=10)$ the same amount of placebo, daily. The supplement and the placebo were given from week 1 until week 10 of the trial, orally. Skin biopsies were taken three times, once at the beginning of the study (week 0), once at half time (week 5) and once at the end of the study (week 10), followed by histological analysis including leucocytes count twice in five vision fields of each slide. No alterations of hair, adnexal structures or skin thickness were found. However, the number of perivascular lymphocytes turned out to be increased at the end of the study in 9 out of 10 animals of the study group, whereas number of lymphocytes in biopsies of the control group where increased only in 4 out of 10 animals. The increase of lymphocytes in the study group suggests an activation of the immune system due to the application of Equi-Strath ${ }^{\bullet}$.
\end{abstract}

\section{Keywords}

Equine, Skin, Biopsies, Plasmolyzed Herbal Yeast, Lymphocytes

\section{Introduction}

Strath ${ }^{\oplus}$ Bio-Strath ${ }^{\oplus}$ is a food supplement known to boost the immunological 
state of the human body. The product is based on a specially produced plasmolyzed herbal yeast with malt, honey and orange syrup which contains 61 vital substances. Its effect of the immune system of human beings and mice was tested in several studies. In 1988, Leslie et al. showed that daily administration of Strath ${ }^{\otimes}$ Bio-Strath ${ }^{\otimes}$ prolonged survival time in old mice, even after infection with Staphylococcus aureus [1] [2]. Since mortality rates were reduced, but there were no changes in morbidity, it was suggested that supplementation of Strath ${ }^{\otimes}$ Bio-Strath ${ }^{\otimes}$ had a stress protectant effect [1]. In a study concerning the mode of action of of Strath ${ }^{\circledast} /$ Bio-Strath $^{\otimes}$ yielding higher survival rates, it could be demonstrated that Strath $\%$ Bio-Strath ${ }^{\odot}$ administration encourages more rapid and efficient mobilization of leucocytes including helper/inducer T-lymphocytes and B-lymphocytes in mice [3] [4]. Similar findings were seen in T-lymphocytes activation in vitro under the stress of low-gravity [5]. The addition of the food supplement partly restored or even increased expression of activation markers on T-cells [6]. These studies indicate an improved capacity of the immune system after long-time administration of Strath ${ }^{\oplus} /$ Bio-Strath $^{\odot}$. Additionally, it is as-

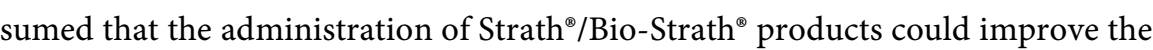
skin quality and hair condition in animals. In this study Equi-Strath ${ }^{\oplus}$, the adapted product for horses was tested. The aim of the study was to understand the effects of Equi-Strath ${ }^{\circledast}$ on the skin and hair condition in the horse.

\section{Material and Methods}

\subsection{Animals}

The experiment was carried out at the Swiss National Stud of Agroscope in Avenches, Switzerland, and included 20 Franches-Montagnes stallions. All horses used for the study showed no obvious health disorders, and were confirmed clinically healthy by a veterinarian at the start of the experiment. The stallions were sexually experienced, aged on average $9.5 \pm 4.5$ years (mean \pm standard deviation), and had a bodyweight of $525 \pm 30 \mathrm{~kg}$ (mean \pm standard deviation).

Animal experimentation was performed following approval from the local animal ethics committee (Etat de Vaud, Service Vétérinaire, approval \#2667.1). The stallions were kept in individual box stalls with straw or wood chip bedding and were fed hay or haylage. In addition, oats, barley, corn and pellets were fed daily. Water and a mineral salt block were available ad libitum. All animals were regularly exercised and were daily turned out into a paddock throughout the study.

\subsection{Experimental Design}

The stallions were randomly divided into two groups, a study group (SG, $n=10$ ) and a control group (CG, $n=10$ ). The stallions of the study group each received a $0.06 \mathrm{ml} / \mathrm{kg}$ bodyweight of Equi-Strath ${ }^{\oplus}$, daily. The control group (CG) received a placebo equivalent in consistency, taste and color as Equi-Strath ${ }^{\circledR}$, at the same quantity daily. The experiment lasted for 10 weeks starting in October 2013. 
Since the administration of Equi-Strath ${ }^{\circledR}$ was very well accepted by the horses, the supplement and the placebo were given, orally, from week one until week ten of the trial.

\subsection{Biopsies}

Three times during the study, biopsies of haired skin were taken of each horse included, once before the beginning (week 0) of the application of Equi-Strath ${ }^{\circledast}$, once at half time (week 5), and once at the end (week 10). Biopsies, $4 \mathrm{~mm}$ in diameter, were taken from the necks of the horses, dorsal under the mane approximately $5-10 \mathrm{~cm}$ from the instep, either on the right or left side. Before performing the biopsies, $1 \mathrm{ml}$ of lidocaine (2\%) was injected subcutaneously and hair was shaven at the biopsy site. Then skin was washed with betadine and ethanol (70\%), three times each. Biopsies were taken using a biopsy punch (KRUUSE Biopsy punch steril $4 \mathrm{~mm}$, Langeskov, Denmark). At the end incisions were stapled with one or two staples each or alternatively, sutured. All 60 skin biopsies were taken without sedation of the horses.

\subsection{Histological Slides}

The biopsies were fixed in $4 \%$ formalin for 48 hours at room temperature. Then they were manually cut parallel to the hair angle and embedded in paraffin according to a standard protocol. Slides of $3 \mu \mathrm{m}$ thickness were cut with a microtome and colored with hematoxylin and eosin (HE). Slide production was performed at the Institute of Veterinary Pathology of the University of Bern.

\subsection{Histological Analysis}

Histological slides of haired skin were examined with magnifications of $5 \mathrm{x}, 10 \mathrm{x}$, 20x, and 40x. Perivascular lymphocytes, plasma cells, eosinophils, mast cells and macrophages in the superficial dermis were evaluated and counted twice within five vision fields on each slide. Within the hair bulb, medulla, cortex, cuticle as well as the dermal papilla could be assessed and thickness could be measured. Along the hair shaft inner and external root sheath thickness was measured as well and compared between the study group and the control.

\subsection{Statistical Analysis}

The nonparametric data of the leucocyte counts at the beginning and the end of the study was analyzed using Wilcoxon Signed-Rank Test (Paired T-Test). Data analysis was conducted using NCSS statistical software (NCSS 2007, LLC, Utah, USA). In addition, a two-sided Z-test of two proportions (success = increase of number of lymphocytes) was performed using the same software.

\section{Results}

All of the 60 histological slides of haired skin were utilizable for analysis. 57 of 
them showed a cut exactly parallel to the hair follicles. Three slides were cut in a slightly sloped angle but still readable. All analyzed skin biopsies showed healthy non-altered skin with small to moderate amounts of leucocytes (Figure 1). In all slides, epidermis, superficial and deep dermis were visible. Occasionally, subdermal fatty tissue was found. On each slide, within the superficial dermis numerous longitudinal sections and fewer cross sections of hair follicles were visible. They were surrounded by unaltered adnexal structures as sebaceous glands and hair musculature ( $M$. erector pili) (Figure 2). Growing stage of all hair follicles were anagen and catagen in October (week 0), catagen to telogen in November (week 5) and telogen in December (week 10). The stages did not differ between the groups. The thickness of epidermis was slightly enhanced in two animals of the control group which also showed locally expanded severe inflammation (animal 5 and 19). Slight hyperkeratosis was also seen in the same samples. Otherwise, there was no difference of the haired skin between the study and the control group. The numbers of mast cells, plasma cells, eosinophils and macrophages, did not show any relation to either intake of Equi-Strath ${ }^{\circledast}$ or time within the study. In nine of ten animals of the study group the number of perivascular lymphocytes was augmented $(2.77 \pm 2.024$ perivascular lymphocytes per vision field (mean \pm standard error)) at the end of the trial (week 10) compared to the beginning (week 0). Numbers of perivascular lymphocytes in the skin of the control group $(1.18 \pm 0$ perivascular lymphocytes per vision field (mean \pm standard error)) at the end of the study were augmented only in four of the ten

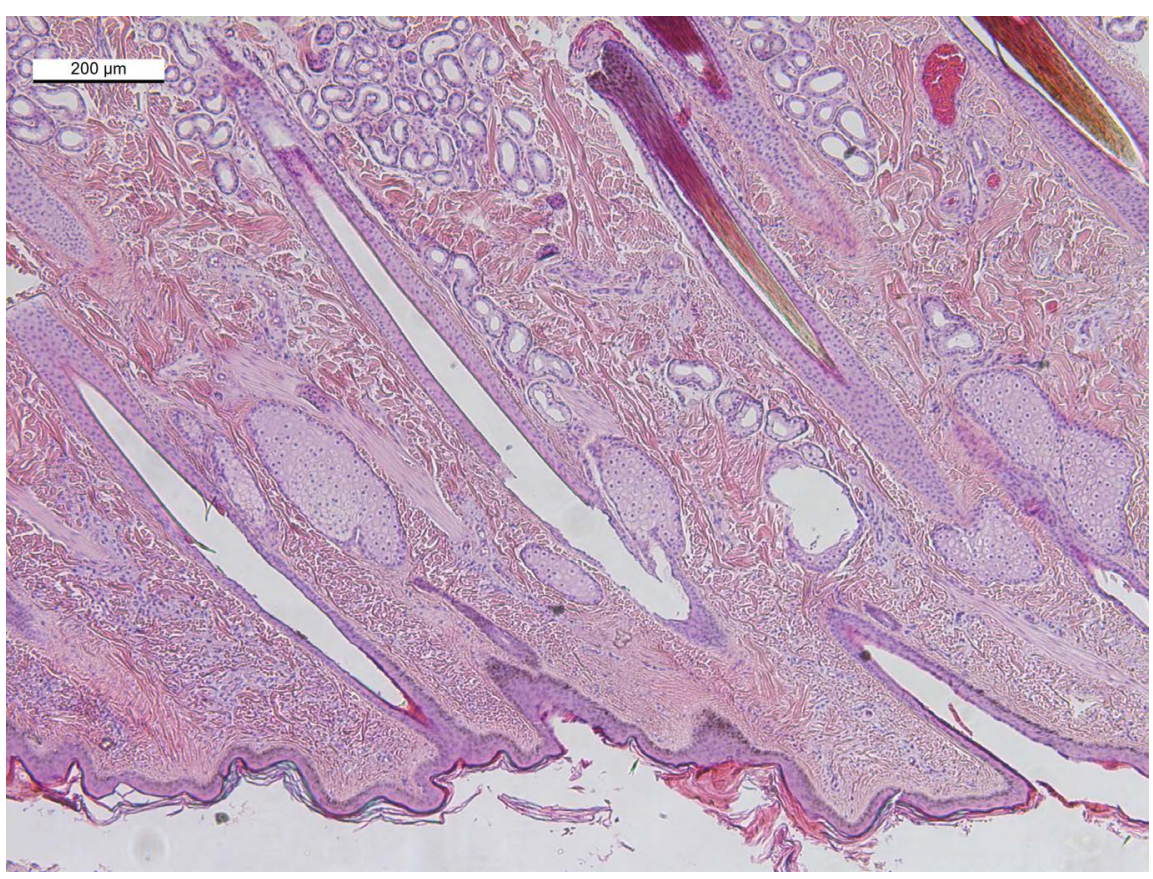

Figure 1. Histological cross section of skin from a horse of the study group at week 5 of the study ( $\mathrm{H} \& \mathrm{E}$ staining). No pathological alterations are visible within the catagen to telogen hair follicles and the adnexal structures including sebaceous glands and hair musculature. 
animals included (Table 1). Comparing the proportions of success, being increased numbers of perivascular lymphocytes, statistical analysis revealed a significant difference between the groups $(p=0.02)$. No significance was shown by Wilcoxon Signed-Rank Test.

\section{Discussion}

Plasmolyzed herbal yeast products have been shown to have a beneficial effect

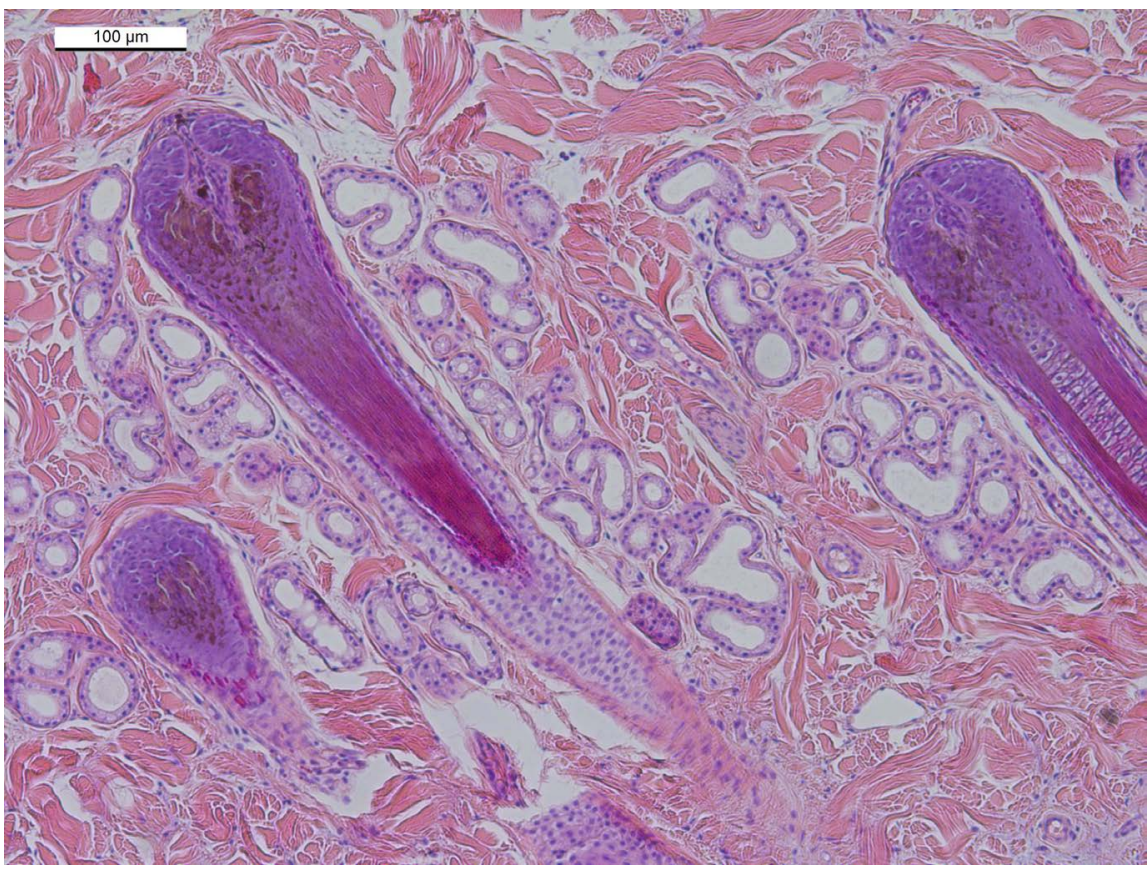

Figure 2. Histological cross section of skin from a horse of the study group (week 0 of the study) showing normal adnexal structures and anagen hair follicles without any pathological alterations (H \& E staining).

Table 1. Mean of perivascular lymphocyte count (absolute numbers in five vision-fields/ slide) at week 0 and week 10 of the study. $\Delta=$ difference between the number of perivascular lymphocytes at the different time points.

\begin{tabular}{ccccccccccc}
\hline & \multicolumn{10}{c}{ Study group (SG, $\boldsymbol{n}=\mathbf{1 0})$} \\
\hline Animal number & 2 & 4 & 6 & 8 & 10 & 12 & 14 & 16 & 18 & 20 \\
Week 0 & 6.2 & 4.6 & 4.4 & 6.8 & 6.1 & 3.8 & 5 & 6.2 & 16.8 & 9.4 \\
Week 10 & 14.8 & 7.6 & 5 & 11 & 7.4 & 4.4 & 4.8 & 8.8 & 22.2 & 10.8 \\
$\Delta$ (SG) & $\mathbf{8 . 6}$ & $\mathbf{3}$ & $\mathbf{0 . 6}$ & $\mathbf{4 . 2}$ & $\mathbf{1 . 3}$ & $\mathbf{0 . 6}$ & $-\mathbf{0 . 2}$ & $\mathbf{2 . 6}$ & $\mathbf{5 . 4}$ & $\mathbf{1 . 4}$ \\
\hline & & \multicolumn{1}{c}{ Control group (CG, $\boldsymbol{n}=\mathbf{1 0})$} & & & & \\
\hline Animal number & 1 & 3 & 5 & 7 & 9 & 11 & 13 & 15 & 17 & 19 \\
Week 0 & 7.8 & 7.6 & 4.6 & 10.2 & 7.4 & 4.4 & 4.8 & 6.6 & 10.6 & 10 \\
Week 10 & 6 & 11 & 10.4 & 10.2 & 7.2 & 4.4 & 4.8 & 8.2 & 9.8 & 13.8 \\
$\Delta$ (CG) & -1.8 & $\mathbf{2 . 4}$ & $\mathbf{5 . 8}$ & $\mathbf{0}$ & $-\mathbf{0 . 2}$ & $\mathbf{0}$ & $\mathbf{0}$ & $\mathbf{1 . 6}$ & $-\mathbf{0 . 2}$ & $\mathbf{3 . 8}$ \\
\hline
\end{tabular}


on the immune system of mice and human beings [1] [2] [3] [4] [7]. Daily application of Equi-Strath ${ }^{\otimes}$ seems to increase numbers of perivascular lymphocytes in horses. The differences between animals can be explained with the individual condition of the animals, although all were of the same breed, same sex, and confirmed clinically healthy by a veterinarian. In the control group two animals (animal 5 and 19) were found to show a higher increase of perivascular lymphocytes compared to the others. Both of them also showed thickening of the epidermis due to dermal inflammation, this activation of the immune system might be the explanation for the increase of perivascular lymphocytes of these animals of the control group.

The overall results in this study show that in the horse, perivascular lymphocytes are slightly increased after ten weeks of Equi-Strath ${ }^{\otimes}$ application, leading to the assumption that the immune system of equines is activated by its daily administration. Supposedly, through the activation of lymphocytes the ability of the immune system to react against bacterial and viral infections is also increased. Whether these are B- or T-lymphocytes should be the subject of additional studies.

Although it was assumed, that Equi-Strath ${ }^{\circledast}$ has a beneficial effect on the hair and skin constitution, no obvious differences in the skin condition otherwise was seen. No difference concerning hair condition or even in the thickness of hair was found between the two groups at the end of the study. The timing of the study coincided with the growth of the winter coat, referring to only one full haircycle, which explains the difference in the growing stages of the hair at the different time points during the study. To determine whether the hair condition would show any changes over time the administration of Equi-Strath ${ }^{\circledast}$ should be prolonged.

\section{Conclusion}

Daily administration of $0.06 \mathrm{ml} / \mathrm{kg}$ bodyweight Equi-Strath ${ }^{\oplus}$ did not affect the skin and hair condition, but resulted in an increase of dermal perivascular lymphocytes, which might reflect a beneficial effect on the immune system of healthy horses.

\section{Acknowledgements}

We would like to thank all who participated in this study, notably the Swiss National Stud Avenches, for disposing its stallions and infrastructures as well as its staff. This work was supported by ISMEquine Research, the School of Agricultural, Forest and Food Sciences (HAFL), and by Bio-Strath ${ }^{\circledR}$ AG.

\section{References}

[1] Leslie, G.B., Frasdillla, J.C., Salmon, G.K. and Sturman, G. (1988) The Effect of Lifetime Treatment with a Herbal Yeast Food Supplement (Bio-Strath ${ }^{\circledast}$ ) on Survival and Infection Resistance in Mice. SWISS MED, 10, 36-38. 
[2] Leslie, G.B., Canham, D. and Frasdilla, J.C. (1989) The Effect of 30 Days Treatment with a Herbal Yeast Food Supplement (Bio-Strath ${ }^{\circledast}$ ) on Infection Resistance in Old Mice. SWISS MED, 11, 30-31.

[3] Joller, P. (1988) Reinforcing Immune Defence against Bacterial Infections Using a Yeast Preparation. SWISS MED, 10, 30-35.

[4] Joller, P. and Aeppli, R.E. (1989) Influence of Prophylactic Administration of a Yeast Preparation (Bio-Strath ${ }^{\oplus}$ ) on the Defence Response. SWISS MED, 11, 66-71.

[5] Joller, P.W., Schwarzenberg, M. and Cogoli, A. (2000) Mode of Action of Plasmolysed Yeast on Lymphocytes under Microgravity Stress Immunological Investigation. Immunological Investigation, 29, 355-364.

[6] Schwarzenberg, M., Joller, P. and Cogoli, A. (2000) Stress-Compensation by a Food Supplement Based on Yeast Plasmolysate in Mitogen Activated T-Lymphocytes under Simulated Low-Gravity. Biological Sciences in Space, 14, 3-8.

https://doi.org/10.2187/bss.14.3

[7] Gmünder, F.K. (1990) Effect of a Herbal Yeast Food Supplement and Long-Distance Running on Immunological Parameters. British Journal of Sports Medicine, 24, 103-112. https://doi.org/10.1136/bjsm.24.2.103 